Review Article

\title{
TUBERCULOSIS PROBLEM IN DAKAHLIA GOVERNORATE, EGYPT
}

\author{
Amina Mostafa Abdel Aal, Noha El-Mashad, Dalia Magdi \\ Department of Clinical Pathology, Mansoura Faculty of Medicine, Egypt.
}

\begin{abstract}
Tuberculosis (TB) is a potentially fatal contagious disease that can affect almost any part of the body but is mainly an infection of the lungs. It has been present in humans since antiquity. In the past, tuberculosis has been called consumption, because it seemed to consume people from within, with a bloody cough, fever, pallor, and long relentless wasting. In Egypt, TB constitutes the second most important public health problem after schistosomiasis. Although Egypt has relatively low levels of TB according to data from the World Health of Organization, $2005: 66 \%$ of TB cases occur among the socially and economically productive age groups of 15 to 54 years. According to Ministry of Health and Population (MOHP), Egypt; tuberculosis control is carried out through 111 chest centers and 39 chest disease hospitals. Treatment failure accounts for $3 \%-5 \%$ of the treatment outcome of new smear positive cases and $13 \%-17 \%$ of retreated cases and this is due to non-compliance to treatment, deficient health education to the patient, poor patient knowledge regarding the disease and diabetes mellitus as co-morbid. The incidence and prevalence of tuberculosis in Egypt has been declining due to increased efforts of the MOHP. Prevalence dropped from 88/100,000 population in 1990 to 24 in 2008, according to data from WHO.
\end{abstract}

Key words: Tuberculosis, Problem, Egypt

\section{INTRODUCTION}

Tuberculosis (TB) is a growing international health concern. It is a big killer among the infectious diseases in the world, despite the use of a live attenuated vaccine and several antibiotics. After years of decline, TB has re-emerged as a serious public health problem worldwide, especially with increased drug resistance among Mycobacterium tuberculosis (MTB) strains which hinders the success of TB control programs. ${ }^{1}$

\section{Interesting facts regarding tuberculosis}

Nearly one percent of the world's population is newly infected with TB each year. Every second, someone in the world is newly infected with TB.

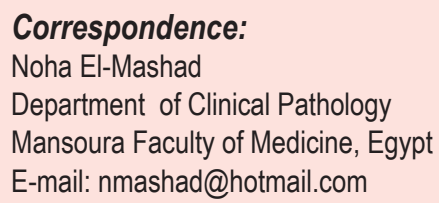

Six to eight million new cases of TB are diagnosed each year. Two hundred million people worldwide, or $10 \%$ of those infected, will develop active TB and be able to infect others for 3 decades if not treated. TB spreads through the air and is highly contagious. On average, a person with infectious TB infects 10-15 others every year. TB kills 8,000 people a day - that is 2-3 million people each year. It kills more people than either AIDS or malaria. In fact, TB is the biggest killer of young people and adults in the world today. In the last 100 years, 200 million people have died of TB. People infected with TB do not necessarily become ill- the immune system creates a barrier around the bacilli that can remain dormant for years. $10 \%$ of infected people (who do not have HIVIAIDS) develop active TB at some point during their lifetime. ${ }^{2}$

Tuberculosis is a contagious disease, like the common cold; it spreads through the air when droplet nuclei are inhaled. The most effective (infective) droplet nuclei tend to have a diameter of 5 um and generated during talking, coughing and 
sneezing. Coughing generates about 3000 droplet nuclei, while talking for 5 minutes generates 3000 droplet nuclei but singing generates 3000 droplet nuclei in one minute. Sneezing generates the most droplet nuclei by far, which can spread to individuals up to 10 feet away. ${ }^{3}$ Each droplet may transmit the disease, since the infectious dose of the disease is very low and inhaling less than ten bacteria may cause an infection ${ }^{4}$. Transmission can only occur from people with active not latent TB. ${ }^{5}$

In the $17^{\text {th }}$ and $18^{\text {th }}$ centuries, TB caused one fourth of the adult's death in Europe. Between 1953 and 1984, the incidence of the disease steadily decreased about 5-6\% every year because of sanitarial improvement, nutrition, ventilation and later because of anti-tuberculous drugs. ${ }^{6}$

Para-aminosalycilic acid (PAS) was discovered in 1940, Isoniazid (INH) in 1952 and Rifampicin in 1960. The introduction of pharmacologic treatment has decreased the incidence of tuberculosis, with predictions of possible eradication. Tuberculosis incidence rates stabilized in most of the world, with increases in African countries and Eastern Europe in recent decades. This tuberculosis reappearance was caused by the AIDS pandemic, emergence of resistant bacilli, human migration patterns, and world poverty. ${ }^{7}$

It is estimated that deaths from TB will increase from 3 million a year currently to 5 million by the year 2050. ${ }^{8}$ Between 2002 and 2020, approximately one billion people will be newly infected, 200 million people will get sick and 36 million will die of TB if proper control measures are not instituted. ${ }^{9}$

\section{Other names of TB}

In the past, tuberculosis has been called consumption, because it seemed to consume people from within, with a bloody cough, fever, pallor, and long relentless wasting. Other names included phthisis (Greek for consumption) and phthisis pulmonalis; scrofula (in adults), affecting the lymphatic system and resulting in swollen neck glands; tabes mesenterica, TB of the abdomen and lupus vulgaris, TB of the skin; wasting disease; white plague, because sufferers appear markedly pale; king's evil, because it was believed that a king's touch would heal scrofula; and Pott's disease, TB of the spine and joints. ${ }^{10}$

\section{Geographic distribution}

Tuberculosis is prevalent in Russia, India, Southeast Asia, sub-Saharan Africa, and parts of Latin America. The highest number of deaths occurred in the Africa region, where HIV has led to rapid growth of the TB epidemic. ${ }^{11}$

In developing countries, there is an annual incidence of 100 to 450 new cases per 100,000 inhabitants, with 2 to 3 million deaths per year, and $75 \%$ of cases affect people between 15 and 50 years of age. Conversely, developed countries have a lower incidence, with 7 to 15 new cases per 100,000 inhabitants and 40,000 deaths per year, the elderly, ethnic minorities and immigrants are chiefly affected. ${ }^{7}$

In 2007, the prevalence of TB per 100,000 people was highest in sub-Saharan Africa, and was also relatively high in Asia. The annual incidence rate varied from 363 per 100,000 in Africa compared to 32 per 100,000 in the Americas. India had the largest total incidence, with an estimated 2.0 million new cases in that year. ${ }^{12}$

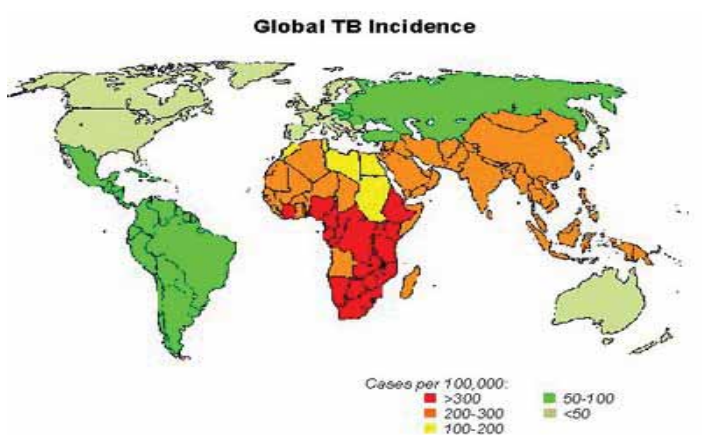

Figure 1. The following ten countries account for the largest number of TB cases among immigrants: Mexico, Philippines, Vietnam, India, China, Haiti, South Korea, Guatemala, Ethiopia, and Peru. ${ }^{13}$

Mycobacterium tuberculosis is the causative agent of most cases of tuberculosis. ${ }^{15} \mathrm{It}$ is a small bacillus that can withstand weak disinfectants and can survive in a dry state for weeks, protected by a complex and hardly penetrable cell wall. Its special structure is the reason for the resistance to external factors and for the inefficient uptake of antibacterial substances. ${ }^{14}$

The rapid and accurate detection of TB is essential for management of patients and public health control. ${ }^{15}$ 
In many countries diagnosis of TB is performed by microscopic examination of a stained sputum smear by the Ziehl-Neelsen (ZN) method. Although easy to perform and specific, it lacks sensitivity, requiring $\geq 10,000$ bacilli per $\mathrm{ml}$ of sputum to become positive. ${ }^{16}$

The gold standard for TB diagnosis is the cultivation of M.tuberculosis. It is much more sensitive than microscopy and it allows recovery of the bacteria for other studies, such as drug susceptibility testing and genotyping. ${ }^{17}$ It can detect 10-100 organisms $\mathrm{per} / \mathrm{ml}$ however, it is time consuming and takes 6 to 8 weeks for the results. ${ }^{18}$

Lack of sensitivity in smear examination, nonspecificity of radiological findings, extended time of M.tuberculosis culture and difficulties in diagnosing paucibacillary; childhood and extra pulmonary tuberculosis have necessitated exploring the utility of immunodiagnosis of tuberculosis for definite diagnosis. $^{19}$

Serological methods are simple, rapid, inexpensive, and relatively non- invasive. They can potentially distinguish between active disease and asymptomatic infection ${ }^{20}$. Many tests are available for diagnosis of TB. Most of these tests are based on the detection of $\lg G, \lg A$ and $\lg M$ antibodies to specific mycobacterial antigen or mixture of antigens. ${ }^{19}$

Molecular diagnosis of tuberculosis has enabled rapid detection of M.tuberculosis complex in clinical specimens, identification of mycobacterial species, detection of drug resistance, and typing for epidemiological investigation. ${ }^{21}$

Many molecular methods have been developed; these methods can potentially reduce the diagnostic time from weeks to days. PCR-based sequencing has become commonly used to identify many mycobacterial species. DNA probes have been widely used for species determination of the most commonly encountered mycobacteria. Highdensity oligonucleotide arrays (DNA micro arrays) also have been applied. ${ }^{22}$

\section{TB problem in Mansoura}

Tuberculosis is one of the important public health problems ${ }^{23}$. It constitutes a major public health problem after HCV and bilharziasis. ${ }^{24}$
Although Egypt is not on the WHO list of 22 high-TB-burden countries, it is considered one of the high-burden countries in WHO's Eastern Mediterranean region. ${ }^{23}$

Tubercular decay has been found in the spines of Egyptian mummies from 3000-2400 BC. ${ }^{25}$ The first evidence that human tuberculosis was present in ancient Egypt came from typical macroscopic osseous changes in human remains, which were in this instance the well preserved Egyptian mummies. ${ }^{26}$

Within the last few years a considerable number of molecular studies have provided evidence for the presence of $M$. tuberculosis complex DNA in ancient skeletal and mummified material. ${ }^{25}$

Tuberculosis is one of the important public health problems in Egypt. However, limited information on the M.tuberculosis genotypes circulating in Egypt is available. ${ }^{26}$

Inadequacies in health data collection and dissemination in Egypt make it unlikely that information obtained on tuberculosis morbidity and mortality will be accurate. ${ }^{27}$

M. tuberculosis was identified as the etiologic organism in $19.7 \%$ of patients with infectious neurologic disease at the Abbassia Fever Hospital in Cairo, Egypt, from 1966 to 1989. This prevalence was second only to that of meningococci, and TB meningitis was the most frequent cause of death at this hospital. ${ }^{28}$

With an estimated TB incidence of 11 new cases per 100,000 people, Egypt has relatively low levels of TB according to 2005 data from the World Health Organization. ${ }^{29}$

The estimated annual risk of infection with TB has declined from $3.5 \%$ in 1952 to $0.24 \%$ in 2006 , meaning that nearly 24 patients per 100,000 get the disease every year and 11 out of those 24 are sputum smear positive. ${ }^{30}$

Every year, the National Tuberculosis Control Program of the Ministry of Health and Population (MOHP) registers over 12,000 new TB patients; more than $50 \%$ of the cases are sputum smearpositive pulmonary TB. On the basis of an annual 
risk of infection of $0.32 \%$, it is estimated that about 8,000 people receive a diagnosis of TB at facilities other than those of MOHP. ${ }^{31}$

Although Egypt has an intermediate level of incidence and mortality (24 and 3 per 100,000 populations respectively), $66 \%$ of TB cases occur among the socially and economically productive age groups of 15 to 54 years. The latest surveillance data obtained in 2006 reveals that, the national population in Egypt is: 71,348,000 and Prevalence rate of TB is $35 / 100,000$ of the population. Multidrug resistant TB is $2.2 \%$ of the new cases \& $38.4 \%$ among re-treatment cases according to a national survey in $2002 .{ }^{2}$

In this locality, a study made by Zaghloul ${ }^{32}$, included 100 cases (86 of them were suspected and 14 were diagnosed as TB). Age distribution of tuberculous cases (17) represented $23.5 \%$ for age $20-30$ followed by $17.7 \%$ for each of ages $1-10,30-40,40-50 \&>60$ years. In contrary, age group 10-20 \& 50-60 was zero percent and 5.9\% respectively. Regarding sex; males represented $76.5 \%$ while females represented $23.5 \%$. Regarding occupation, among tuberculous cases, workers and non-employed persons constituted $29.4 \%$ for each followed by professional $(23.53 \%)$, then farmers (11.76\%) and house wives (5.88\%). There was a statistically highly significant increase of rural residence $(76.47 \%)$ over urban residence $(23.53 \%)$.

Another study made by Dawood ${ }^{33}$, searched for renal tuberculosis. The study included 60 cases (35 of them were suspected to be urinary tuberculosis "Group I" and 25 were under anti-tuberculous therapy "Group II") and 20 control "Group III". The most frequent age ranged from 20 to 30 years $(31.6 \%)$ followed by age group from $30-40$ years (26.6\%), the age group from $40-50$ and $50-60$ years represent $(15 \%)$ for each age. The last frequent ages range were $>60$ and 10-20 years represent $5 \%$ and $6.6 \%$ respectively. Male frequency $(63.3 \%)$ was higher than female frequency $(36.7 \%)$. The most common presenting complaints were dysuria $(86.7 \%)$, renal colic $(61.7 \%)$ urinary frequency $(56.7 \%)$, positive past history of urinary TB $(46.7 \%)$, loss of weight $(28.3 \%)$, hematuria and fever $(26.7 \%$ for each), loss of appetite (25\%), night sweating $(15 \%)$, kidney transplantation (6.7\%), infertility and past history of pulmonary TB (3.3\% for each).
Tuberculosis in renal transplant recipient is a serious problem which has high morbidity and increased mortality. ${ }^{34}$ In Egypt, the incidence of TB among 700 kidney recipients in urology and nephrology center in Mansoura University were 4\% and this was considered high compared to other areas. $^{35}$

In Mansoura, Egypt a 5 years study (1998 - 2003) included 3790 specimens from different sites of the body suspected to have TB (sputum, urine, semen, endometrial biopsy, pleural fluid, pus, CS etc.) was performed, the patient's age ranged from 3 to 70 years (male represent $64 \%$ and females $36 \%$ ). The most frequent cases were young adults. The results revealed TB cases in $6.2 \%$. Positive samples for TB were $9.6 \%$ in sputum, $4.2 \%$ in urine, $3.1 \%$ in semen and $8.6 \%$ in endometrial biopsies, while other body fluids were negative for TB. ${ }^{15}$

\section{Tuberculosis control in Egypt}

The incidence and prevalence of tuberculosis in Egypt has been declining due to increased efforts of the MOHP. Prevalence dropped from 88/100,000 population in 1990 to 24 in 2008, while incidence dropped from 34 in 1990 to 19 in 2008 according to data from $\mathrm{WHO}^{36}$

Tuberculosis control in Egypt is carried out through 111 chest centers and 39 chest disease hospitals with 6351 hospital beds. Tuberculosis control activities are integrated through the primary health care system. The health staff working in these units is highly qualified due to the sustainable plan of human resource development. The WHO Directly Observed Treatment strategy (DOTs) was implemented starting from 1996 and became available to all patients in MOHP chest clinics by August 2000. ${ }^{30}$

The World Health Organization strongly advocates the use of directly observed therapy, short course (DOTs) elsewhere for drug-sensitive cases. This simple procedure means that the patient must be seen to swallow their medication under the eye of a trained (not necessarily medically) supervisor. Along with the proper prescription of drugs, WHO believes that DOT monitoring, which ensures that patients take the prescribed medications for the appropriate periods of time, will greatly reduce drug resistant TB. ${ }^{30}$ 
Egypt is classified as one of the 36 worldwide countries having achieved the global targets in both case detection and treatment success under DOTS. ${ }^{11}$ In 2008, the case detection rate of positive cases in Egypt was 78\% (global target is 70\%) and treatment success rate was $89 \%$ (global target is 85\% (Ministry of Health and Population, 2010).

Treatment failure is a serious problem facing some national tuberculosis control programs. Significant risk factors for treatment failure in Egypt were irregularity of treatment non-compliance to treatment, deficient health education to the patient, poor patient knowledge regarding the disease and diabetes mellitus as co-morbid condition. In Egypt, treatment failure accounts for $3 \%-5 \%$ of the treatment outcome of new smear positive cases and $13 \%-17 \%$ of re-treatedcases. ${ }^{37}$

Drug-resistant strains of $M$. tuberculosis pose serious public health problems. Multiple drug resistance and low cure rates are the most important problems facing TB control efforts in Egypt. There is a little information regarding the distribution of strains and the development of drug resistance, particularly in major population regions outside the immediate vicinity of Cairo. ${ }^{38}$

The development of drug resistance in the population has increased the possibility that TB may once again become an incurable disease..$^{18}$ Resistance to drugs is due to particular genomic mutations in specific genes of MTB. To date, nine genes are known to be linked to resistance to firstline anti-TB drugs; katG, inhA, aphC, and kasA for INH resistance, rpoB for RIF resistance, rpsL and rrs for streptomycin resistance, embB for EMB resistance, and pncA for pyrizinamide resistance. Resistance to multiple drugs is the consequence of an accumulation of mutations. ${ }^{39}$

In Mansoura the rate of drug resistance was $32.7 \%$, with a higher prevalence of resistance in patients who had received previous antituberculous treatment $(70 \%)$ than new patients $(30 \%)$, according to the study which was carried out on 153 tuberculous patients (83 males and 70 females) with positive Bactec 460 culture for acid fast bacilli. Those patients were selected from Mansoura University Hospitals and Mansoura Chest Hospital.Genotypic analysis was done for rifampicin resistant specimens and $\mathrm{INH}$ resistant specimens to detect mutations responsible for drug resistance by PCR amplification of rpoB gene for rifampicin resistant cases and KatG gene for isoniazid resistant cases. Finally, DNA sequencing was done for detection of mutation within $\mathrm{rpoB}$ and KatG genes. Genotypic analysis of RIF resistant cases revealed that $86.9 \%$ of RIF resistance were having rpoB gene mutation versus $13.1 \%$ having no mutation with a high statistical significant difference between them. Direct sequencing of Kat $\mathrm{G}$ gene revealed point mutation in92.3\%and the remaining $7.7 \%$ had wild type KatG i.e. no evidence of mutation with a high statistical significant difference between them. ${ }^{40}$

According to Badran, 2007 drug resistance of TB is higher among rural residence than urban residence. INH shows the highest resistance, followed by rifampicin and ethambutol. The lowest resistance is for streptomycin.

\section{CONCLUSION}

Although Egypt is not on the WHO list of 22 high-TB-burden countries, it is considered one of the high-burden countries in WHO's Eastern Mediterranean region, where TB constitutes the second most important public health problem after schistosomiasis.

The incidence and prevalence of tuberculosis in Egypt has been declining due to increased efforts of the MOHP. Prevalence dropped from 88/100,000 population in 1990 to 24 in 2008, while incidence dropped from 34 in 1990 to 19 in 2008 according to data from $\mathrm{WHO}$.

\section{REFERENCES}

1. Palomino JC. Newer diagnostics for tuberculosis and multi-drug resistant tuberculosis: Curr Opin Pulm Med, 2006;12:172-8. World Health Organization. STOP-TB Partnership. The Global MDR-TB \& XDR-TB Response Plan 2007

2. World Health Organization. Global Tuberculosis Control Report, 2006 - Annex 1 Profiles of highburden countries.

3. Nicas M, Nazaroff WW, Hubbard A. Toward understanding the risk of secondary airborne infection: emission of respirable pathogens. J Occup Environ Hyg, 2005;2:143-54. 
4. Kumar, Abbas, Fausto, Mitchell. Robbins Basic Pathology(8th ed), Saunders Elsevier, 2007;51622.

5. Gomez JE, McKinney JD. Consumed in the city observing tuberculosis at century's end. J Clin Invest, 2004:484.

6. André A Figueiredo, Antônio $M$ Lucon MD, Urogenital Tuberculosis: Update and Review of 8961 Cases from the World Literature Rev Urol, 2008;10:207-217.

7. Jun Yue T, Shi W, Xie J, Li Y, Zeng E, Wang H. Mutation in the rpoB gene of multidrug-resistant Mycobacterium tuberculosis isolates from China.J. of Clinical Microbiol. 2003;41:2209-2212.

8. Boshoff HIM, Reed MB, Barry CE and Mizrahi VTB or Not TB: How Mycobacterium tuberculosis may evade drug treatment Cell, 2003;139-145.

9. Barun Mathema, Natalia E Kurepina, Pablo J Bifani, Barry N Kreiswirth Molecular Epidemiology of Tuberculosis: Current Insights. Clinical Microbiology Reviews, 2006;19:658-85.

10. Rajagoplan $S$ and Yoshikawa TT. Tuberculosis in long-term facilities. Infect. Control Hosp Epidemiol, 2000;21:611-6.

11. World Health Organization (2009). "Epidemiology". Global tuberculosis control: epidemiology, strategy, financing. pp. 6-33.

12. Jackson C, Roesel D. Epidemiology of TB: Primary Care Tools for the Management of TB. ethno MED 2010.

13. Ryan KJ, Ray CG Sherris Medical Microbiology (4th ed.). McGraw Hill., 2004;247-9.

14. Hoffmann $C$, Leis $A$, Niederweis $M$, Jürgen $M$, Plitzko, Harald E. Disclosure of the mycobacterial outer membrane: Cryo-electron tomography and vitreous sections reveal the lipid bilayer structure. Proceedings of the National Academy of Sciences USA, 2008.

15. Abd El-aal MARapid, Recent Laboratory Diagnosis of Tuberculosis and Its Drug Susceptibility. Project in Mansoura University (5 years study) Faculty of Medicine, Mansoura, Egypt 2006.

16. Angeby KA, Hoffner SE, Diwan VK. Should the bleach microscopy method be recommended for improved case detection of tuberculosis. Literature review and key person analysis. Int J Tuberc Lung Dis, 2004;8:806-15.
17. Torres A and Caminero J. Nonconventional and new methods in the diagnosis of tuberculosis. Eur Respir J, 2005;26:339-350.

18. Skims, Soura, Srinagar, Kashmir, Acharya Shri Chander. Newer methods for the laboratory diagnosis of tuberculosis. JK-Practitioner, 2001;8:266-69.

19. Bahatia AS, Satish Kumar, Harinath BC Immunodiagnosis of tuberculosis. Indian Journal of clinical Biochemistry, 2003;18:1-5.

20. Allied Sci Advances in serology for diagnosing TB in HIV infected. Indian J Chest Dis.,2005;47:31-37.

21. Cheng VC, Yew WW, Yuen KY. Molecular diagnostics in tuberculosis. Eur $\mathrm{J}$ Clin Microbiol Infect Dis, 2005;24:711-20.

22. Kumar $P$, Nath $K$, Rath $B$, Sen M K, Vishalakshi $P$, Chauhan DS, Sreenivas V. Visual Format for Detection of Mycobacterium tuberculosis and M. bovis in Clinical Samples Using Molecular Beacons.J. Mol. Diagn., 2009;11:430-38.

23. Helal ZH, Ashour MS, Eissa SA, Abd-Elatef G, Zozio T, Khan Ml. Unexpectedly High Proportion of Ancestral Manu Genotype Mycobacterium tuberculosis Strains Cultured from Tuberculosis Patients in Egypt. Journal of Clinical Microbiology,2009;47:2794-2801.

24. Zaher $\mathrm{H}$. Tuberculosis situation in Egypt. The fifth International Conference of Microbiology, AIDS and Emerging Infectious Disease. Cairo, Egypt, 1996.

25. Sola C, Zink AR, Reischl U, Grabner W, Rastogi $\mathrm{N}$, Wolf $\mathrm{H}$, et al Molecular identification and characterisation of Mycobacterium tuberculosis complex in ancient Egyptian mummies. Int $\mathrm{J}$ Osteoarchaeol, 2004;14:404-13.

26. Zink AR, Sola C, Reischl U, Grabner W, Rastogi N, Wolf $\mathrm{H}$ et al.. Characterization of Mycobacterium tuberculosis Complex DNAs from Egyptian Mummies by Spoligotyping . Journal of Clinical Microbiology., 2003:41:359-67.

27. Ashour M, Helal Z H, Eissa SA, Abd-Elatef G, Zozio T, Babapoor S, Rastogi N, et al. Unexpectedly High Proportion of Ancestral Manu Genotype Mycobacterium tuberculosis Strains Cultured from Tuberculosis Patients in Egypt. Journal of Clinical Microbiology, 2009;47:2794-2801.

28. World Health Organization Global tuberculosis control. WHO Report 2000. Geneva. 
29. Abbadi SH, Cooksey RC, Woodley CL, Sikes D, Wasfy M, Crawford J, et al. Characterization of Mycobacterium tuberculosis Complex Isolates from the Cerebrospinal Fluid of Meningitis Patients at Six Fever Hospitals in Egypt. J Clin Microbiol, 2002;405:1651-55.

30. World Health Organization TB Rates Worldwide,.2008.

31. Health Profile: Egypt. United States Agency for International Development, 2008.

32. National Tuberculosis Control Program, annual report. Ministry of Health and Population, Egypt, 2006.

33. Zaher HA, Tag El Din MA, van Maaren PJM. Tuberculosis control and intersectoral collaboration: the experience of Egypt. National Tuberculosis Control Programme, Cairo, Egypt, 2008.

34. Zaghloul MH. Updated technology in diagnosis and drug susceptibility of Mycobacterium tuberculosis. Thesis for the M.D degree in Clinical Pathology. Central Library Faculty of Medicine, Mansoura University, Egypt, 1998.

35. Dawood M Value of modern microbilogical technique in diagnosis of renal tuberculosis. Thesis for the Master degree in Clinical Pathology. Central Library Faculty of Medicine, Mansoura University, Egypt, 2000.

36. Lenk S. Oesterwitz H, Scholz D. Tuberculosis in cadaveric renal allograft recipients. Eur Urol, 1988;14:484-86.

37. Hamid MH. Impact of tuberculosis on outcome of kidney transplantation. Urology and Nephrology Center. M.D. Thesis for the Master degree in
Clinical Pathology. Central Library Faculty of Medicine, Mansoura University, Egypt, 1995.

38. Ministry of Health and Population-Egypt Current situation in Egypt towards achieving The Millennium Development Goals MDG.,2010.

39. Morsy AM, Zaher HH, Hassan MH, Shouman A. National Tuberculosis Control Programme, Ministry of Health and Population, Cairo, Egypt.. Predictors of treatment failure among tuberculosis patients under DOTS strategy in Egypt. East Mediterr Health J, 2003;9:689-701.

40. Abbadi S H, Rasheed H G, Morlock G P, Woodley C L, El Shanawy O, Cooksey R C. Characterization of IS6110 restriction fragment length polymorphism patterns and mechanisms of antimicrobial resistance for multidrug-resistant isolates of Mycobacterium tuberculosis from a major reference hospital in Assiut. Egypt. J Clin Microbiol, 2001;39:2330-34.

41. Annelies VR, Warren R, Mshanga I, Jordaan AM, Spuy GD, Richardson M, et al. Analysis for a limited number of gene codons can predict drug resistance of Mycobacterium tuberculosis in a high-incidence community. J ClinMicrobiol, 2001;39:636-41.

42. Abd El-aal MA, Abd El-Ghaffar H, El-Mashad $N$, Badran E, Fathy A. Genotypic detection of rifampicin and isoniazid resistant Mycobacterium tuberculosis strains by DNA sequencing: a randomized trial. Ann Clin Microbiol Antimicrob, 2009;8:4.

43. Badran E. Molecular diagnosis of drug resistant tuberculosis. M.D Degree thesis. Fac Med Mansoura University, 2007. 